\title{
A General Technique for Characterizing X-Ray Position Sensitive Arrays*
}

E. Dufresne, R. Bruning, M. Sutton

Center for the Physics of Materials

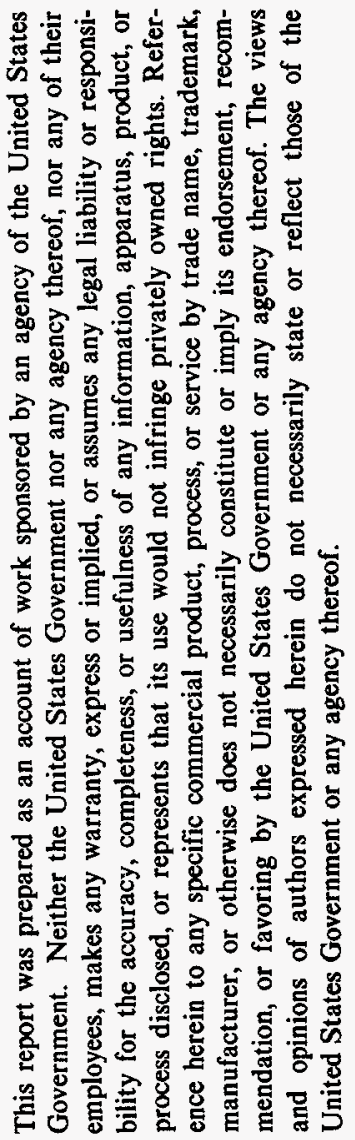

jw
G.B. Stephenson

IBM Research Division

Thomas J. Watson Research Center

Yorktown Heights, NY 10598
March, 1994

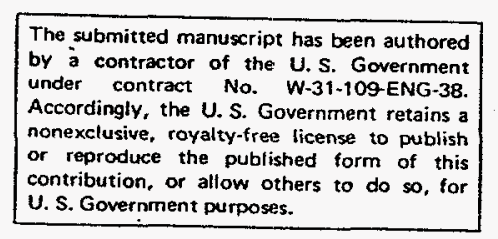

Department of Physics

McGill University

Montreal, Canada H3A 2T8

B. Rodricks

Experimental Facilities Division

Advanced Photon Source

Argonne National Laboratory

Argonne, IL 60439

Fo 15193

OSTI

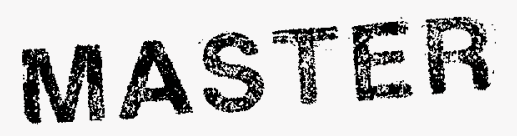

DISTPIBUTION OF THIS DOCUMENT IS UNLMITED BS

*This work supported by the U.S. Department of Energy, BES-Materials Sciences, under contract no. W-31-109-ENG-38 


\section{DISCLAMMER}

Portions of this document may be illegible in electronic image products. Images are produced from the best available original document. 


\title{
A general technique for characterizing x-ray position sensitive
} arrays.

\author{
E. Dufresne, R. Brüning, M. Sutton \\ Center for the Physics of Materials, Department of Physics, McGill University, Montreal, \\ Canada H3A 2T8
}

B. Rodricks

Advanced Photon Source, Argonne National Laboratory, g700 South Cross Avenue, Argonne, Mlinois 60439

\author{
G. B. Stephenson
}

IBM Research Division, Thomas J. Watson Research Center, Yorktown Heights, NY 10598

(January 26, 1994)

\begin{abstract}
We present a general statistical technique for characterizing $x$-ray sensitive linear diode arrays and CCD arrays. We apply this technique to characterize the response of a linear diode array, Princeton Instrument model X-PDA, and a virtual phase CCD array, TI 4849 , to direct illumination by $\dot{x}$-rays. We find that the response of the linear array is linearly proportional to the incident intensity and uniform over its length to within $2 \%$. Its quantum efficiency is $38 \%$ for $\mathrm{Cu} K_{\alpha} \mathrm{x}$-rays. The resolution function is evaluated from the spatial autocorrelation function and falls to $10 \%$ of its peak value after one pixel. On the other hand, the response of the CCD detecting system to direct $\mathrm{x}$-ray exposure is non-linear. To properly quantify the scattered $\mathrm{x}$-rays, one must correct for the non-linearity. The resolution is twapiofels along the
\end{abstract}


serial transfer direction We characterize the noise of the CCD and propose a model that takes into account the non-linearity and the resolution function to estimate the quantum efficiency of the detector. The quantum efficiency is $20 \%$.

PACS:?????? 


\section{INTRODUCTION}

The advent of high intensity $x$-ray synchrotron sources coupled with the development of $\mathrm{x}$-ray position sensitive detectors has allowed us to study time-resolved Noh-equilibrium dynamics in solid-state systems. For example, linear diode arrays have been used to study the isothermal crystallization of metallic glasses by measuring structure factors with a time resolution of a few milliseconds [1] and were used to study the early stage dynamics of a continuous phase transition in $F e_{3} A l$ [2] and ordering in $C u_{3} A u$ [3]. Two dimensional position sensitive detectors like a CCD array have been used to study the dynamics of a first order phase transition in $C u_{3} A u$ with coherent x-rays [4-6] or to study strain kinetics in $I n_{x} G a_{1-x} A s$ quantum wells [7].

To obtain meaningful quantitative data from a position sensitive detector, one must know whether the response of the detector is linear to the incident number of photons, verify that this response is uniform over the detector area; measure the noise the resolution function of the detector and its quantum efficiency.

In this paper, we show how one characterizes the response of a position sensitive detector by comparing the measured noise to the noise expected from Poisson counting statistics. Similar techniques have been used in the past [8], but neglected to consider the effect of spatial correlation between pixels on the calibration. Significant spatial correlation exists between neighboring pixels for typical position sensitive detectors, and we show how one can extract the resolution function from the correlation function. We discuss in detail how this correlation affects the characterization of the detector. These effects are important for coherent diffraction, when one needs a resolution of the order of the pixel size [4-6].

In the first section, we give a general treatment for characterizing one and two dimensional detectors and show a simplified technique for a single linear detector. The second section describes the characterization of a $x$-ray sensitive photodiode array. The third section describes a more general technique used for a charged coupled detector (CCD) detector, a Texas Instrument TI 4849 virtual phase chip. 


\section{DESCRIPTION OF THE TECHNIQUE}

Solid state position sensitive detectors are normally made of an array of Si photodiodes, which are used as integrating detectors for x-rays. A photon in the range of $5-20 \mathrm{keV}$ will generate thousands of electron-hole pairs which are then collected on the the Si diode capacitance. (For $\mathrm{Si}, 3.6 \mathrm{eV}$ is required to create an electron hole pair [10].) After a preset exposure time, the charge collected is measured, amplified, digitized and finally cleared for the next integration. The detected signal $S$, measured in analog to digital units (ADU), is proportional to the accumulated charge, and depends on the gain setting of the amplifier. To characterize a detector, one must test the detector's linearity, noise and uniformity, measure its resolution and quantum efficiency.

To test uniformity, one normally illuminates the detector with a spatially uniform source of light or $\mathrm{x}$-rays. To generate this uniform source, we may scatter a beam of monochromatic $\mathrm{X}$-rays onto an amorphous sample like a piece of polyimid (kapton) [9] or polystyrene and place the detector far away from the sample. To measure the detector's uniformity, one verifies that $S$, the detected signal, is uniform over the detector area. For further discussion, we have defined a few terms of interest in Table 1.

To test linearity, one varies the incident number of photons $n_{i}$ and the integration time independently to see whether or not the total integrated number of photons is the only relevant quantity. One must find a function that relates $S$ to $n_{i}$ so that $S=f\left(n_{i}\right)$. In

general, $f$ hot linear. Therefore to linearize the detector's signal, one would apply the inverse of $f$ to $S$. Once the data is linearized, one also wants to know the signal to noise in these new units. A second relationship can be established: $\sigma_{i}^{2}=g\left(n_{i}\right)$ where $\sigma_{i}^{2}$ is the variance in linearized units.

For a single linear detector, a simple technique can be used. This technique assumes that detected x-ray photons obey Poisson distribution. Assuming that $S$ is proportional to $n_{d}$, taking a time average of this uniform scattering by averaging several scans gives $<S>_{t}=k<n_{d}>_{t}$, where $k$ is the proportionality constant which corresponds to the num- 
ber of ADU per detected photons. From $\sigma_{S}^{2}$ and $\sigma_{d}^{2}$, the time averaged variances of $S$ and $n_{d}$, follow the relation $\sigma_{S}^{2}=k^{2} \sigma_{d}^{2}$. Taking the ratio of $\sigma_{S}^{2}$ over $<S>_{\ell}$, recalling that $\left.\sigma_{d}^{2}=<n_{d}\right\rangle_{\imath}$ for a Poisson distribution, one finds that

$$
\frac{\sigma_{S}^{2}}{<S>_{t}}=\frac{k^{2} \sigma_{d}^{2}}{k<n_{d}>_{t}}=k
$$

So by taking a ratio of the time averaged variance of the detected signal over its time average, one determines the ratio of ADU to detected photons for each pixel. $n_{d}$ will be smaller than the incident number of photons on the detector $\left(n_{i}\right)$ by a factor equal to the quantum efficiency. For a complete treatment, $\alpha$ can be calculated by measuring $\boldsymbol{n}_{i}$ with a detector with near unit efficiency.

One can also measure the spatial variations in $k$ to test the spatial uniformity of the detector. In order to determine if these fluctuations are meaningful, we need to know what is the error on $k$ expected from Poisson noise. The uncertainty in the determination of $k$ depends on the number of measurements N. From standard error propagation analysis, $\sigma_{k}$, the uncertainty on the ratio of two correlated random variables $\sigma_{S}^{2}$ and $\langle\dot{S}\rangle_{t}$ is given by $[10]$

$$
\frac{\sigma_{k}^{2}}{k^{2}}=\frac{\sigma_{\sigma_{S}^{2}}^{2}}{\sigma_{S}^{4}}+\frac{\sigma_{\langle S\rangle}^{2}}{\langle S\rangle^{2}}-2 \frac{\operatorname{cov}\left(\sigma_{S}^{2},<S>\right)}{\left.\sigma_{S}^{2}<S\right\rangle}
$$

where the covariance $\operatorname{cov}\left(\sigma_{S}^{2},\langle S\rangle\right)=\overline{\sigma_{S}^{2}\langle S\rangle}-\overline{\sigma_{S}^{2}} \overline{\langle S\rangle}$. Here $\sigma_{\sigma_{S}^{2}}^{2}$ is the variance of $\sigma_{S}^{2}$. It can be shown for a Poisson distribution that $\frac{\sigma_{S}^{2}}{\sigma_{S}^{2}}=\frac{2}{N-1}+\frac{1}{N n_{d}}, \frac{\sigma_{\langle S\rangle}^{2}}{\langle S\rangle^{2}}=\frac{1}{N n_{d}}$ and $2 \frac{\operatorname{cov}\left(\sigma_{S}^{2},\langle S\rangle\right)}{\sigma_{S}^{2}\langle S\rangle}=\frac{2}{N n_{d}}$. Replacing all the terms in Eq (2), we find

$$
\frac{\sigma_{k}}{k}=\sqrt{\frac{2}{N-1}}
$$

From Eq. (3), we see that by taking large $N$, we can measure our calibration constant accurately. The error is caused by the relative error on the measured variance. Note that the covariance term cancels exactly the dependence on $n_{d}$. (This is not true for a Gaussian distribution where the covariance is zero. Then $\frac{\sigma_{k}}{k}=\sqrt{\frac{2}{N-1}+\frac{\sigma_{d}^{2}}{N n_{d}^{2}} \text {.) }}$ 
A note of caution for this treatment must be made. This technique assumes that each pixel is independent from its neighbors. If this is not true, the interpretation of $k$ is not simply the number of digital units per photons. This extra correlation reduces the variance. It is discussed below how this affects $k$.

The resolution function is normally measured by illuminating the detector with a source, which is smaller than the pixel size. For a CCD or a linear array, often this would require collimating the $x$-ray beam through a pinhole with a diameter of only a few micrometers and scanning the pinhole over the detector's area. Because this requires an intense $\mathrm{X}$-ray source and a translation system, this technique may be difficult to apply.

In order to evaluate the resolution function, we wered a simple tool that can be used to characterize any spatially sensitive detector. We used the spatial autocorrelation of a random signal $S(\vec{r})$ as a way to probe the resolution of the detector. The autocorrelation function is defined by:

$$
C(\vec{\Delta})=\frac{\left\langle S(\vec{r}) S(\vec{r}+\vec{\Delta})>_{\vec{r}}-<S(\vec{r})>_{\vec{r}}^{2}\right.}{\left\langle S(\vec{r})^{2}>_{\vec{r}}-<S(\vec{r})>_{\vec{r}}^{2}\right.}
$$

where the bracket refers to a spatial average $\langle S(\vec{r})\rangle_{\vec{r}}=\sum_{\vec{r}} S(\vec{r}) / N_{p}$. Here, $N_{p}$ is the total number of pixels in the region correlated and $\vec{r}$ is a vector indexing the multidimensional array of the detector. Two limiting cases in this problem may be calculated easily. For large enough $\vec{\Delta}, S(\vec{r})$ and $S(\vec{r}+\vec{\Delta})$ are independent. Using $\langle S(\vec{r})\rangle=\langle S(\vec{r}+\vec{\Delta})\rangle=\mu$ for a uniform incident beam, $\left\langle S(\vec{r}) S(\vec{r}+\vec{\Delta})>_{\vec{r}}=\mu^{2}\right.$ and $\mathrm{C}(\vec{\Delta})=0$. For $\vec{\Delta}=0, C(0)=1$. If the signal between neighbors is not independent, one finds $0<C(\Delta)<1$. Next we show how the width of the correlation function can be related to the autocorrelation function of the resolution function.

A model developed for the shot noise process, described in [11], may be used to calculate the resolution function and its effect on the measured noise. The model describes a stochastic process that is created by a random superposition over time or space of a known response function $h(x)$, where $x$ can be a spatial or a temporal coordinate. For example, in a scintillator, $h(x)$ is the pulse shape associated with the detection of a photon. The measured 
detector's signal, $\mathrm{S}\left(x_{0}\right)$ at some coordinate $x_{0}$, is simply a sum of these disturbances centered at randomly spaced times or positions $x_{i}$ i.e.

$$
S\left(x_{0}\right)=\sum_{i=1}^{n_{d}} h\left(x_{i}-x_{0}\right)
$$

In our experiment, $n_{d}$ is the number of detected photons, and is sampled from a Poisson distribution. The $x_{i}$ are chosen from a univariate distribution because the signal is spatially uniform. It can be shown that the response of the system can be written as [11]:

$$
\begin{gathered}
<S>=n_{d} \int h(x) d x \\
\sigma^{2}=n_{d} \int h(x)^{2} d x \\
\Gamma(\Delta) \equiv<S(0) S(d x)>-<S(0)>^{2}=n_{d} \int h(x) h(x+\Delta) d x
\end{gathered}
$$

where $\langle S\rangle$ is the spatially areraged response, $\sigma^{2}$ is the variance of the detector signal, $\Gamma(\Delta)$ is the spatial autocorrelation function and $n_{d}$ the number of random Poisson events.

If $h(x)$ is a delta function, we recover Eq. (1) from Eq. (6-7). If the response has a finite width, we see that the interpretation of the ratio as a calibration constant is not true anymore, because correlations are introduced due the correlation function of the response function in Eq. (8). By measuring the spatial autocorrelation function, we can invert Eq. (8) to evaluate the resolution function. So by simply illuminating the detector with a spatially uniform beam, we can evaluate both the uniformity of the detector and its resolution function.

\section{CHARACTERIZATION OF A LINEAR POSITION SENSITIVE DETECTOR}

The detector characteristics are given in Table II. The photodiode array contains 2048

rectangular pixels $25 \mu \mathrm{m}$ wide, $2.5 \mathrm{~mm}$ high. Its resolution is quoted as 1.5 pixel ${ }^{5}$ wide so there will be some correlation between pixels as in Eq. (8). It is operated at $-40^{\circ} C$, cooled by a Peltier stage, to reduce the dark electronic noise. The array is operated by a PI 
ST1000 controller. The data is transferred to a IBM PC 386-AT through a custom designed I/O board from PI. Software is provided with the package to control the data acquisition parameters, store the data and visualize the data. Scans can be accumulated by a 32 bits register and pixels can be grouped to increase the scan rate while sacrificing the spatial resolution.

Figure 1a) shows the results of our statistical technique for the linear array. The time averaged signal, the time averaged variance and the ratio of variance over mean are displayed every 20 pixels on the array for two different $x$-ray energies, 6.93 and $8.05 \mathrm{keV}$. The first data set was obtained by scattering $6.93 \mathrm{keV} x$-rays onto the $(1 / 2,1 / 2,1 / 2)$ order-disorder peak of $\mathrm{Fe}_{3} \mathrm{Al}$ below $T_{c} .100$ scattering patterns were averaged. Note that the mean is quite smooth (i.e well defined to within $0.3 \%$ ) but that the fluctuations on the variance are substantial ( $14 \%$ ). The relative error on the mean is $\frac{1}{\sqrt{N n_{d}}}$ while the error on the variance goes as $\sqrt{\frac{2}{N-1}}$, where $N$ is the number of measurements. The error bars on the ratio were calculated from Eq. (3). A least.square fit of the ratio to a constant yields $k=0.682$ and a $\chi^{2}$ of 1.1. The ratio is constant over the detector within the wide error.bars based on 100 measurements.

Figure 1b) was obtained by placing the detector in front of a $\mathrm{x}$-ray tube with a $\mathrm{Cu}$ target . and a Si (111) monochromator set on $\mathrm{Cu} K_{\alpha}$. To determine $k$ more accurately, 8000 patterns were averaged. The beam covered approximately half of the array, and one can see small tails on the average, likely due to diffuse scattering from the slits. Note the logarithmic mean and variance axes. The signal varies over 3 order of magnitude but the ratio is independent of signal level. On the tails, the signal level is low and the dark noise contributes to some of the variance observed. To account for this extra variance, a measured dark variance of 1.1 $A D U^{2}$ was subtracted to the variance before calculating the ratio. A least-square fit of the ratio to a constant between pixel 650 to 1400 gives $k=0.792$ and a $\chi^{2}$ of 2 . This residual is due to fluctuations on the array's response which have a rms amplitude of $2 \%$. Note that only because we averaged a large number of scans we are able to see these fluctuations. If signal variations between pixels of the order of a few $\%$ are important, then these fluctuations 
have to be taken into account by using different linearization constant for each pixel.

The number of electron-hole pairs per photon is proportional to energy. In Fig. 1, one can also notice that the ratio is a function of energy so that $\frac{k(8.05)}{k(6.93)}=\frac{0.792}{0.682}$ is equal to the ratio of energies. Let us now measure the spatial correlations.

First, we define the variable $s(i)=S(i)-\langle S(i)\rangle_{\ell}$ which is introduced to remove some systematic variations on the data due to the beam profile. Here, $i$ is the pixel index which corresponds to the one dimensional vector $\vec{r}$ in Eq. (4). Figure 2 shows the spatial autocorrelation function of $s(i)$ as calculated from $C(\Delta)=\frac{\langle a(i) \cdot(i+\Delta)\rangle_{i}-\langle a(i)\rangle_{i}^{2}}{\left\langle\Delta(i)^{2}\right\rangle_{i}-\langle\Delta(i)\rangle_{i}^{2}}$ where \langle\rangle$_{i}$ refers to a spatial average. The error bar on this correlation function is proportional to $\sqrt{1 / N}[12]$ where $N$ is the number of data points averaged. Figure 2 shows $C(\Delta)$, with $C(1)=0.215 \pm 0.001, C(2)=0.024 \pm 0.001$ and and $C(3)=0.000 \pm 0.001$.

As discussed in section II, the resolution function is evaluated from Eq. (6-8), using the following model for $h(x)$. When a photon hits a pixel, it causes a signal $h_{0}$ in this pixel and a signal $h_{1}$ in its nearest neighbors. Replacing the integrals by sums in Eq. (6-8), the averaged signal is $n_{d}\left(h_{0}+2 h_{1}\right)$. The variance is $n_{d}\left(h_{0}^{2}+2 h_{1}^{2}\right)$ and the correlation function function for $\Delta=1$ is $n_{d}\left(2 h_{0} h_{1}\right)$.

From this model, we see that:

$$
\begin{aligned}
& k=\frac{\sigma_{S}^{2}}{<S>_{t}}=\frac{\left(h_{0}^{2}+2 h_{1}^{2}\right)}{\left(h_{0}+2 h_{1}\right)}=0.792 \\
& C(\Delta=1)=\frac{\left(2 h_{0} h_{1}\right)}{\left(h_{0}^{2}+2 h_{1}^{2}\right)}=0.215
\end{aligned}
$$

We can see that $k$ is not just the number of ADU per photon, but depends on the actual shape of the response function. Solving for $h_{0}$ and $h_{1}$, one finds $h_{0}=0.956, h_{1}=0.108$. Since we are interested in the number of photons detected $n_{d}$, the number of interest for our calibration will be $\left(h_{0}+2 h_{1}\right)=1.17$. From Eq. (6), one gets $n_{d}$ by dividing the signal by this number. Since we can now measure $n_{d}$, we can estimate the quantum efficiency.

This measurement was performed by collimating the incident $x$-rays with a $1.3 \mathrm{~mm}$ hole centered on the middle of a pixel. We first measured the integrated signal by the detector. 
We then measured the signal with a scintillator placed behind the pinhole. We find that $\alpha=38 \pm 2 \%$ for $8.05 \mathrm{keV}$ x-rays.

The quantum efficiency should go as the the transmission through a $250 \mu \mathrm{m}$ Be window times the transmission through a $1 \mu m \mathrm{SiO}_{2}$ overcoat times the fraction of absorbed x-rays by $\mathrm{Si}$ in the electron diffusion length $t_{S i}$.

$$
\alpha \approx e^{-\mu_{B e} \rho_{B e} t_{B e}} e^{-\mu_{S_{S i O}} \rho_{S_{i O}} t_{S_{i O}}}\left(1-e^{-\mu_{S i} \rho_{S i} t}\right)
$$

where the mass absorption coefficients $\mu$ are $1.1,36.4,64.7 \mathrm{~cm}^{2} / \mathrm{g}$ for $\mathrm{Be}, \mathrm{Si}$ and $\mathrm{SiO}_{2}$ respectively [13] at $8.05 \mathrm{keV}$, and the densities are $1.85,2.32,2.21 \mathrm{~g} / \mathrm{cm}^{3}$ respectively. The $50 \mu \mathrm{m}$ diffusion length is given by the manufacturer [14]. This yields a quantum efficiency of 50 $\%$. The measured quantum efficiency and this simple calculation agrees relatively well given the simplicity of the model.

\section{CHARACTERIZATION OF A CCD ARRAY}

The CCD detecting system has been described in detail previously [15-18]. In brief, a CCD is an array of photodiodes converting the energy of an incident photon into a large number of electron-hole pairs, proportional to the incident number of photons. This charge is stored in a potential well during a fixed exposure time, and then read by a series of parallel row transfers and serial pixel-to-pixel transfers and finally is amplified and digitized.

Two modes of operation of solid-state detector (like a CCD array) have been used previously: direct $x$-ray illumination incident on the detector or a CCD optically coupled to a $x$-ray phosphorescent material. We chose direct illumination of the CCD array because we needed the finest spatial resolution achievable with the CCD determined by the pixel dimension. For our coherent $x$-ray experiments $[4,6]$, we chose a TI 4849 chip, with virtual phase architecture, containing $390 \times 584$ square pixels of $22.4 \mu \mathrm{m}$. This choice was justified by the large depletion depth of the TI $4849(12 \mu \mathrm{m})$ and its sufficient resolution. This large depletion depth increases the efficiency under direct $\mathbf{x}$-ray illumination. 
To characterize the detector, we used an amorphous scatterer to produce a spatially uniform beam covering the whole detector area [9]. Figure 3 shows our experimental set up. We used the high brilliance wiggler beamline X25 at NSLS, set at $7 \mathrm{keV}$ with a $\mathrm{Si}$ (111) monochromator. An ion chamber was used to monitor the incident intensity on the sample. The beam was scattered with a piece of polystyrene. The detector was centered at a scattering angle of $2 \Theta=23^{\circ}$ and placed $1.04 \mathrm{~m}$ from the center of rotation.

We controlled the incident number of photons $n_{i}$ by detuning the monochromator and varied the integration time from 1 to 240 s. To measure $n_{i}$, we used a scintillation detector placed at the CCD position and calibrated the scintillator response in term of incident intensity on the sample, measured by the incident beam ion chamber $\left(I_{\text {mon }}\right)$. A simple linear least-square fit of the form $n_{i}=A I_{\text {mon }}$ yields a good fit with $A=0.266 \pm 0.001$ and a $\chi^{2}$ of 1.7. The data are within $1 \%$ of the fit. Because it was difficult to measure at the same time the CCD response and the scintillation signal, we estimated $n_{i}$ by the monitor signal. The detector was masked with a $4 \mathrm{~mm}$ circular aperture. The highest count rate achieved was 1 photon $/(22.4 \mu m)^{2} /$ sec.

In order to calculate $\left\langle S>_{t}\right.$ and $\sigma_{S}^{2}$, the time averaged mean and variance for each pixel, we exposed the CCD for a given integration time and collected several full frame scattering patterns (up to 50). To properly digitize the signal, the detector electonics applies a bias voltage to the signal. A dark pattern must be taken after a set of exposures to subtract this offset voltage from the data.

In figure 4 as an initial calibration, $\frac{\sigma_{S}^{2}}{\langle S\rangle}$ versus $n_{i}$ is shown for several exposures. Contrary to Fig. 1, $k$ depends on the incident intensity. We were quite surprised to see a non-linear relationship, because previous results showed a linear response to within $0.5 \%$ [15-18] with visible light. Because the detector is not linear, we cannot use the same technique used for the linear diode array. We must generalize the treatment to include the non-linearity and correct it as discussed in section II. Let us now study the non-linearity in more detail. 


\section{A. Non-linearity}

First, we found that taking a time average or a spatial average is quantitatively equivalent G) both for the mean and the variance. This is expected for uniform Poisson noise. As a time saving feature, we thus took a spatial average of a single uniform scattering pattern. Figure 5 shows the mean of the CCD response over a region of $100 \times 100$ pixels $(S)$ versus the expected number of photons that would be detected by a single pixel with a scintillator $\left(n_{i}\right)$. In this figure, the incident number of photons is estimated by $n_{i}=\frac{0.266 I_{\text {mon }} \delta t(22.4 \mu m)^{2}}{\pi(2 \mathrm{~mm})^{2}}$, where $\delta \mathrm{t}$ is the exposure time, $I_{m o n}$ is the monitor count during exposure and the other numbers yields the total number of pixels contained in the $4 \mathrm{~mm}$ circular aperture. Two curves are displayed, the first calibration before our measurements (squares) and the last calibration obtained 10 days later at the end of the run (circle). Except for a small difference, the curves are very similar. For the purpose of calibration, we used the first calibration because of its larger range of detector response.

The response of the detector depends only on the incident number of photons $n_{i} \propto I_{\text {mor }} \delta t$. This is seen in Fig. 5, since all the data points with an equal product $I_{\text {mon }} \delta t$ lie on the same curve, although they differ widely in exposure times and incident number of photons. Since $n_{i}$ is the only independent variable, we fitted the above data to several functions to get a smooth relation that linearizes the CCD response as discussed in section II. The solid line in Fig. 5 is a power law least square fit of the form $n_{i}=f^{-1}(S)=0.35 S^{0.84}$, which was found to have the lowest $\chi^{2}$. Although it is not continuous at the origin, it describes the data better than the other fits tried. It was used to linearize the data in our coherent scattering measurements. In our data treatment, we apply this function to the measured intensity $S$.

In order to determine the uncertainty in the linearized signal $f^{-1}(S)$, we need to evaluate the time averaged variance per pixel under uniform scattering. Since we found that a spatial average was equivalent to a time average, the square of the uncertainty was obtained by measuring a variance over a region of the detector from a single measurement.

Figure 6 shows the variance of $n_{i}$ in a region of $100 \times 100$ pixels versus the mean after 
linearization of the raw data by our power law transformation. The solid line is a simple Poisson law $\left(\sigma_{i}^{2}=n_{i}\right)$, while the dotted line is $\sigma^{2}=1.63 n_{i}$. We have more variance than expected from a Poisson law, by about $63 \%$. The observed extra variance will be explained in detail in the discussion. Let us now study the spatial uniformity of the detector.

\section{B. Spatial uniformity in response}

Figure $7(a, b)$ show averages of a single exposure taken over columns or rows of the detector. Rows 110 to 480 and columns 10 to 380 were used for the averages, which cover most of the detector. The mean is uniform in both directions except for small overall drifts of typically around $1 \%$.

Figure 8 shows a bitmap of the time averaged variance $\sigma_{S}^{2}$, displayed using an inverted grey scale. The variance array was calculated with 23 frames exposed for 1 second each. The response is relatively uniform over the whole detector area, except for a few bad columns in the parallel transfer direction, along the $y$ axis. This uniformity is shown in Fig: 9, where the average of the variance over row 420 to 520 is displayed as a function of the horizontal position. In this figure, the slight monotonous decrease of about $12 \%$ is seen but is simply due to inhomogeneities of the incident beam. For this data only, the detector was placed closer, at $295 \mathrm{~mm}$ from the sample, in order to increase the intensity on the detector and this created a small non-uniformity along $2 \theta$. When the detector is rotated by $90^{\circ}$, the non-uniformity rotates also by the same angle confirming a slight non-uniformity in the beam.

The pixels in column ${ }^{5} 26-27,72-73,181-182,208-209$, have a much lower variance than a typical pixel as seen in Fig. 8 and 9. In these columns the variance is lower than its typical value by a factor ranging between 40 and $80 \%$, while the mean is however quite smooth. (see Fig. 7(a,b)) By simply looking at the time average, one would conclude erroneously that all pixels have the same response. To fully characterize the detector, one must also measure the time averaged variance. 
We see that some of the pixels are less noisy than a typical pixel. A possible explanation for this lower variance may be that there is extra smearing around these pixels which reduces the variance. Let us now study the resolution.

\section{Resolution}

For our coherent $x$-ray experiment, it is important to check for any correlation between neighboring pixels. Our speckle pattern should be around 1-2 pixels wide [4], which may be difficult to resolve. Figure 10 shows a typical autocorrelation function as calculated from Eq. (4). Two curves are shown in the figure, $C(\Delta, 0)$ (vertical bars) and $C(0, \Delta)$ (squares) where the first index refers to the columns and the second to the rows of the CCD. The lag $\Delta$ is measured in units of pixels. $C(\Delta, 0)$ is distinguishable from $C(0, \Delta)$ by its characteristic slower decay: After a few pixels, the correlation function decays to zero as discussed previously. The correlation decays in two pixels along $\mathrm{x}$ but decays in one pixel along y. This shows a lower resolution along the serial transfer direction of the CCD than the parallel transfer direction. One should mention that this correlation is due to the detector and is not beam dependent since after rotating the detector by $90^{\circ}$, one finds that the correlation persist more strongly along the rows.

The correlation coefficient are non-zero for the nearest and next nearest neighbor of $C(0,0)$, i.e. $C( \pm 1,0), C( \pm 1, \pm 1), C(0, \pm 1), C( \pm 1, \mp 1) . C(1,0)=0.52 \pm 0.05, C(0,1)=$ $0.12 \pm 0.01, C(1,-1)=0.07 \pm 0.02$ and $C(1,1)=0.07 \pm 0.02$. There are only 4 independent correlation coefficients since $C(\vec{\Delta})=C(-\vec{\Delta})$, as seen from the definition of the correlation. function in Eq. (4). The resolution is better in $y$, i.e along the columns the correlation falls to $12 \%$ after one pixel. Along the rows, the correlations decays to $52 \%$. This is likely due to the readout process. No dependence of $C$ on $n_{i}$ is observed.

Our resolution function should have a range of interaction of $1-2$ pixels. Using the simplest model possible, we choose $\mathrm{h}(\mathrm{r})$ to be symmetric and given by: 


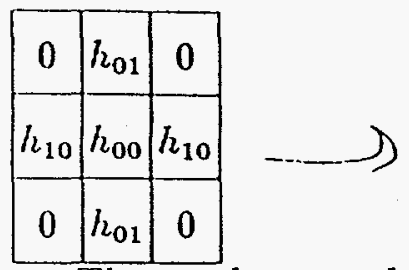

Then, the correlation function of this disturbance can be calculated and gives $\Gamma(0,0)=\left(h_{00}^{2}+2 h_{10}^{2}+2 h_{01}^{2}\right) n_{d}, \Gamma(1,0)=\left(2 h_{00} h_{10}\right) n_{d}$ and $\Gamma(0,1)=\left(2 h_{00} h_{01}\right) n_{d}$. We choose to normalize the integral of $h(r)$ to unity, assuming that one photon will be distributed amongst several pixels so that

$$
h_{00}+2 h_{10}+2 h_{01}=1
$$

Dividing the previous correlation by $\Gamma(0,0)$, we find:

$$
\begin{aligned}
& C(1,0)=\frac{2 h_{00} h_{10}}{h_{00}^{2}+2 h_{10}^{2}+2 h_{01}^{2}}=0.52 \\
& C(0,1)=\frac{2 h_{00} h_{01}}{h_{00}^{2}+2 h_{10}^{2}+2 h_{01}^{2}}=0.12 .
\end{aligned}
$$

Solving Eq. (12-14), we find $h_{00}=0.563, h_{01}=0.042, h_{10}=0.177$. As expected, we find that a large portion of the signal goes to the nearest neighbors along the serial transfer direction.

\section{DISCUSSION}

The previous sections characterized the non-linearity of the detector. We did not discuss its source. Our main concern has been that one of the amplifiers in the detecting system might not be linear. During our experiment, we tried to change the gain on our differential amplifier and also replaced the amplifier but we did not obtain a linear response. We still suspect some electronics to cause this non-linear response since the physical process of creating electron-hole pairs should be proportional to the incident intensity.

Some questions were left unanswered in the previous sections. One of these questions is why do we have extra variance in Fig. 6. To understand quantitatively this extra variance, we propose a model which combines the smearing effect due to the resolution function and the quantum efficiency of the detector. 


\section{A. Smearing due to the resolution function}

If we now use the resolution function as calculated previously, and replace it in Eq. $(6,7)$, one finds the contrast, defined by the variance over the square of the detected mean equal to

$$
\frac{\sigma_{d}^{2}}{\left\langle n_{d}\right\rangle^{2}}=\frac{h_{00}^{2}+2 h_{10}^{2}+2 h_{01}^{2}}{n_{d}}=\frac{0.38}{n_{d}} .
$$

The contrast in this case is smaller than what one expects from Poisson noise by a factor 0.38. This model shows that because our response function smears the signal into several pixels, the variance is reduced. Since the model predicts a smaller variance than Poisson noise, this model is insufficient to explain our extra variance.

\section{B. Quantum efficiency}

Let us now show how the smearing effect discussed previously, the non-linear response of the detector and a quantum efficiency smaller than one explains our extra variance mentioned in Fig. 6. Recalling that $n_{d}$ is the the number of photons detected by the CCD, one would expect $n_{d}$ to follow a Poisson law if each pixel was independent, but since the pixels are coupled,

$$
\sigma_{d}^{2}=K<n_{d}>
$$

where $K=0.38$ is a smearing factor as derived in Eq. (15). From a Taylor's expansion of our linearization function, one can show that:

$$
\sigma_{i}^{2}=\sigma_{S}^{2}\left(d n_{i} / d S\right)^{2}
$$

where $\sigma_{i}^{2}$ is the variance of the linearized data $n_{i}$ and $\sigma_{S}^{2}$ is the variance for the raw data. Since the detector has a quantum efficiency $\alpha$ lower than one, expanding Eq. (16) and replacing $n_{d}$ by $\alpha n_{i}$, one gets

$$
\sigma_{d}^{2}=\alpha^{2} \sigma_{i}^{2}=K \alpha<n_{i}>
$$


Recalling that for our linearization function, $\frac{d n_{i}}{d S}=\frac{B\left\langle n_{i}\right\rangle}{\langle S\rangle}$ and substituting Eq. (17) into Eq. (18), one gets:

$$
\frac{\sigma_{S}^{2}}{\langle S>}=\frac{K<S>}{\alpha<n_{i}>B^{2}}
$$

where $B=0.835$. This equations includes three different contributions in the ratio: the smearing factor, the non-linear behavior and a quantum efficiency. It explains qualitatively why the measured ratio is non-linear, (see Fig. 4) since $\frac{\langle S\rangle}{\left\langle n_{i}\right\rangle}$ is non-linear. This equation gives us also a way to calculate the efficiency by fitting the measured CCD response to Eq: (19).

Figure 4 shows the ratio of $\frac{\sigma_{S}^{2}}{\langle S\rangle}$ versus $n_{i}$. The solid line is a least square fit of Eq. (19) with $\alpha=0.24$. At $7 \mathrm{keV}$, the calculated absorbed fraction of $\mathrm{x}$-rays in the depletion region, is given by $\left(1-e^{-\mu_{s i} \rho_{S i} t}\right)$ where $\mu_{S i}=92.9 \mathrm{~cm}^{2} / \mathrm{g}[19], \rho_{S i}=2.32 \mathrm{~g} / \mathrm{cm}^{3}$ and $\mathrm{t}=12 \mu \mathrm{m}$. This yields a quantum efficiency near $23 \%$ in good agreement with the result of our model.

Our extra variance in Fig. 6 is caused by the extra factor $K / \alpha$. We can see from the equation below that the variance has a prefactor $\mathbb{K} / \alpha$; i.e, a factor 1.5 , which explains our perceived extra variance.

$$
\sigma_{i}^{2}=\frac{\sigma_{d}^{2}}{\alpha^{2}}=\frac{K<n_{d}>}{\alpha^{2}}=\frac{K<n_{i}>}{\alpha}
$$

\section{CONCLUSION}

To obtain quantitative information from a current state of the art position sensitive detector, one must carefully characterize its response. We have shown that we can characterize a position sensitive detector by measuring means, variances, and spatial autocorrelation functions and compare these functions to noise expected for a Poisson distribution. The linear diode array is linear and uniform to $2 \%$. Its resolution, evaluated from the spatial autocorrelation function, falls to about $10 \%$ of the peak value after one pixel. Its quantum efficiency is $38 \%$ for $\mathrm{Cu} K_{\alpha}$ x-rays. We have shown that the response of our CCD detecting 
system to a direct exposure of $x$-rays is non-linear and must be corrected with an appropriate transformation. The response is uniform except for a few bad columns where the noise is smaller than the noise of a typical pixel. One must look at a time averaged variance to notice this effect since the mean is uniform. The resolution is 2 pixels along the serial transfer direction and 1 pixel along the parallel transfer direction. We have estimated the quantum efficiency of the detector to be around $24 \%$ by using a model including the non-linearity of the detector and its resolution function. The linearization of our data appeared to introduce a variance larger than Poisson counting statistic, but this is explained by our model. 


\section{REFERENCES}

[1] S. Brauer, J.O. Ström-Olsen, M. Sutton, Y.S. Yang, A. Zaluska, G.B. Stephenson and U. Köster, Phys. Rev. B, 45, 7704 (1992)

[2] B. Park, G.B. Stephenson, S.M. Allen, K.F. Ludwig Jr., Phys. Rev. Lett., 68, 1742 (1992)

[3] K.F. Ludwig Jr., G.B. Stephenson, J.L. Jordasa-Sweet, J. Mainville, Y.S. Yang and M. Sutton, Phys. Rev. Lett., 61, 1859 (1988)

[4] M: Sutton et al, Nature, 352, 608 (1991)

[5] M. Sutton et al, NSLS Annual Report, BNL 52317, 293 (1991)

[6] E. Dufresne et al, NSLS Annual Report, BNL 52371, 381 (1993)

[7] R. Clarke, W. Dos Passos, W. Lowe, B.G. Rodricks and C. Brizard, Phys. Rev. Lett. , $66,317,(1991)$

[8] T Gao, Y. Li, J. Rousseau, K Linliu and B Chu, Rev. Sci. Instrum., 64, 390 (1993)

[9] J. Mainville, $\mathrm{PhD}$ thesis, McGill University, (1992)

[10] W.R. Leo ,Techniques for Nuclear and Particle Physics Experiments, Springer-Verlag, p75-106, 1987

[11] B.R. Frieden,Probability, Statistical Optics, and Data Testing, 2nd ed., Springer Verlag, $\mathrm{p} 218-228,1991$.

[12] W.H. Press, B.P. Flannery, S.A. Teukolsky and W.T. Vetterling, Numerical recipes in C, Cambridge University Press, p503, 1988

[13] Nuclear Data Center, online database at Brookhaven National Laboratory.

[14] EG\&G Reticon, technical information sheet on the RL2048S Solid State Line Scanner 2048 elements, 345 Potrero Avenue, Sunnyvale, California 94086-4197. 
[15] B. Rodricks and C. Brizard, Nucl. Inst. A, 311, 613 (1992)

[16] C. Brizard and B. Rodricks, Rev. Sci. Instrum., 63, 803 (1992)

[17] R. Clarke and B. Rodricks, Rev. Sci. Instrum., 60, 2280, (1989)

[18] B. Rodricks, R. Clarke, R. Smither and A. Fontaine, Rev. Sci. Instrum., 60, 2586 (1989)

[19] D. Vaughan, X-ray data booklet, Lawrence Berkeley Laboratory, Technical Information Department, Berkeley, California 94720 (1986). 


\section{FIGURES}

FIG. 1. a) time averaged mean, variance, $\frac{\text { variance }}{\text { mean }}$ versus pixel number at $6.93 \mathrm{keV}, \mathrm{N}=100$. The error bars for the mean, variance and ratio are calculated from formulas discussed in section II.

b) $8.05 \mathrm{keV}$ data, $\mathrm{N}=8000$

FIG. 2. Spatial autocorrelation of the signal $S$ for the linear diode array

FIG. 3. Experimental set up.

FIG. 4. $\frac{\sigma_{S}^{2}}{S}$ versus versus the incident intensity on the detector $n_{i}$, where $\sigma_{S}^{2}$ is a spatial variance calculated in a region of 100 by 100 pixels and $S$ is the mean. We take a spatial average on a single frame since it is equivalent quantitatively to a time average. A linear detector would yield a constant independent of the incident intensity. The solid line is a least square fit discussed later in the text.

FIG. 5. Spatial average of the CCD signal $(S)$ over a region of 100 by 100 pixels versus the expected number of photons detected by a single pixel with a scintillator $\left(n_{i}\right)$. Two data sets are shown: the furst calibration displayed with squares and the second calibration 10 days later. The solid line is a least square fit to the first calibration with $n_{i}=0.35 S^{0.84}$. This function is applied to the measured data to linearize it. The detector response was within $75 \%$ of its saturation value for all points.

FIG. 6. This figure shows the spatial variance of the linearized data measured in a region of 100 by 100 pixels versus the mean. One would expect the variance $\sigma_{i}^{2}$ to go as $n_{i}$ in the case of a simple Poisson law (solid line). This is the case for a scintillator. The dotted line is $1.63 n_{i}$, which exceeds Poisson law by $63 \%$. We seem to have extra variance after linearization of the data when we compare the variance to Poisson law. 
FIG. 7. (a) Integrating over one dimension, summing a single row. The sum is shown as a function of the index of the row. The sum is calculated using pixels in columns 10 to 380 .

(b) Integrating over one dimension, summing a single column. The sum is shown as a function of the index of the column. The sum is calculated using pixels in rows 20 to 520 . The data are uniform to within $1 \%$ in both directions.

FIG. 8. A greyscale of a time averaged variance showing pixels included in row 20 to 520 and column 10 to 380 is shown. 23 exposures of one seconds were averaged. An inverted grey scale is used to display the data. The spatial average and standard deviation of the variance are shown. Note that the variance is relatively uniform except for a few pixels in certain columns having a much lower variance than a typical pixel. The time average mean would not show any untypical behavior for these columns but the variance is a more sensitive test.

FIG. 9. Here is an average of 100 pixels of the time averaged variance array along a column between row 420 and 520 versus the column index. The pixels in column 26-27,72-73;181-182, 208-209, have a much lower variance than a typical pixel by a factor ranging between 40 and $80 \%$.

FIG. 10. This figure shows two perpendicular slices of the space autociorrelation function $C(\vec{\Delta})=\left\langle S(\vec{r}) S(\vec{r}+\vec{\Delta})>_{\vec{r}}\right.$ of our uniform scattering data. $C(\Delta, 0)$ (error bars) and $C(0, \Delta)$ (square) are shown, which are respectively correlation functions along the serial transfer direction and the parralel transfer direction. The vertical bars are the standard deviations of the measurement ( $s d=0.01$ ). The correlation decays to 0 in about two pixels along $x$, the serial transfer direction and in one pixel along $y$, the parallel transfer direction. 


\section{TABLES}

\section{TABLE I. Definitions}

S

$n_{i}$

$n_{d}$

$\alpha$

$<>_{t}$

$\sigma_{f}^{2}$

digital signal measured by the detector.

number of photons incident on the surface of the detector as measured by a reference detector having near unity quantum efficiency. number of photons detected by the detector. quantum efficiency of the detector defined as $\alpha=\frac{n_{d}}{n_{i}}$ time average of variable f. $\langle f\rangle_{t}=\frac{1}{N} \sum_{i=1}^{N} f\left(t_{i}\right)$ time averaged variance of f. $\sigma_{f}^{2}=\frac{1}{N-1} \sum_{i=1}^{N}\left(f\left(t_{i}\right)-<f>_{t}\right)^{2}$

\section{TABLE II. Linear position sensitive array characteristics}

PDA

Pixel Dimension

Specified resolution

Window

Amplifier Gain

Dynamic Range

Readout time

Readout noise

Dynamic Range

Integration Time
EG\&G Reticon 2048SAU-822, 2048 pixels

$25 \mu m$ wide, $2.5 \mathrm{~mm}$ high.

1.5 pixels wide.

Direct exposure to x-ray trough a $250 \mu \mathrm{m}$ in $\mathrm{Be}$ window $1300 \mathrm{e} / \mathrm{ADU}$

65000:1

$4 \mu s / p i x e l, 8 \mathrm{~ms}$ for whole array.

$<1.2$ counts rms

65000:1

$8 \mathrm{~ms}$ to two hours. 

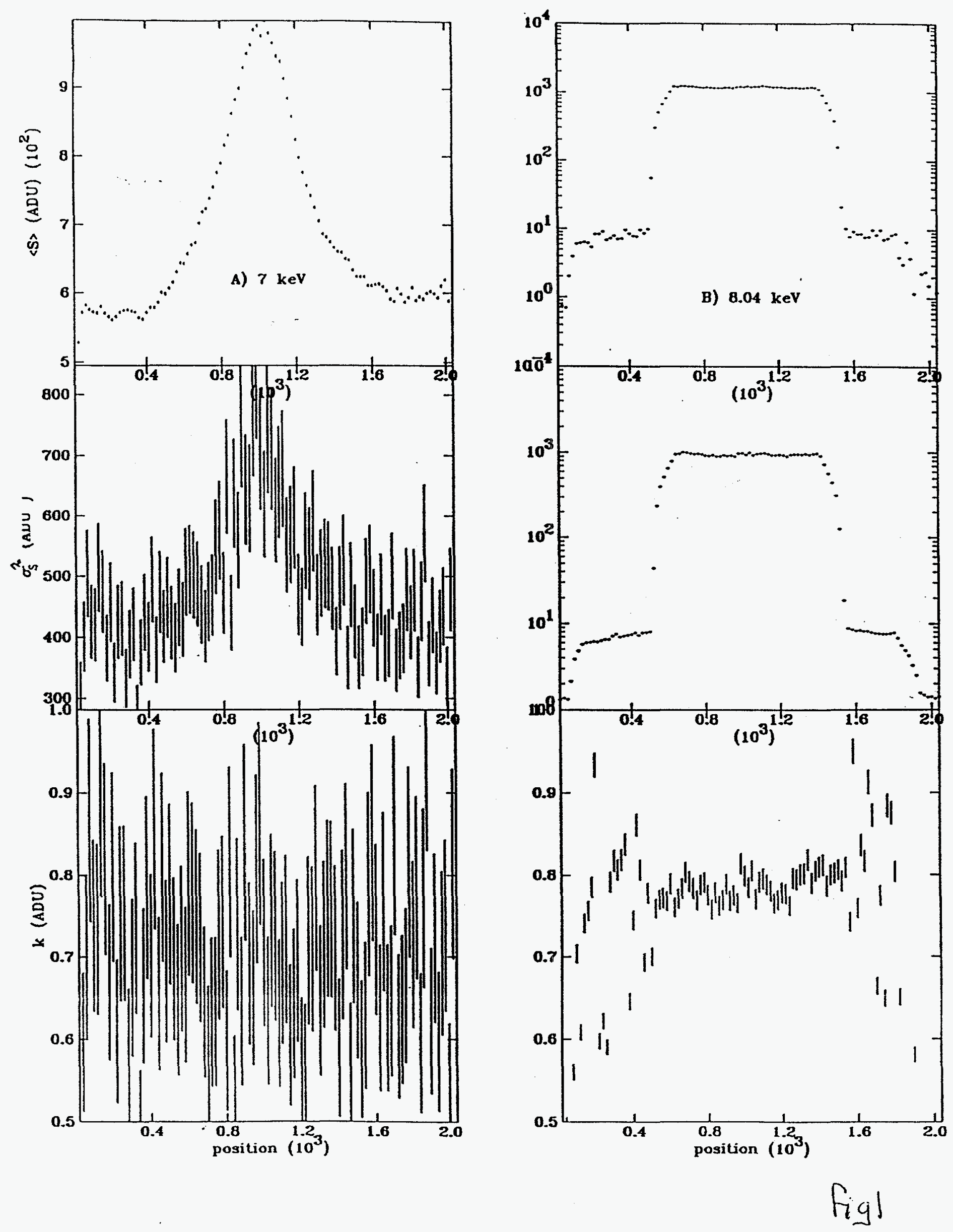


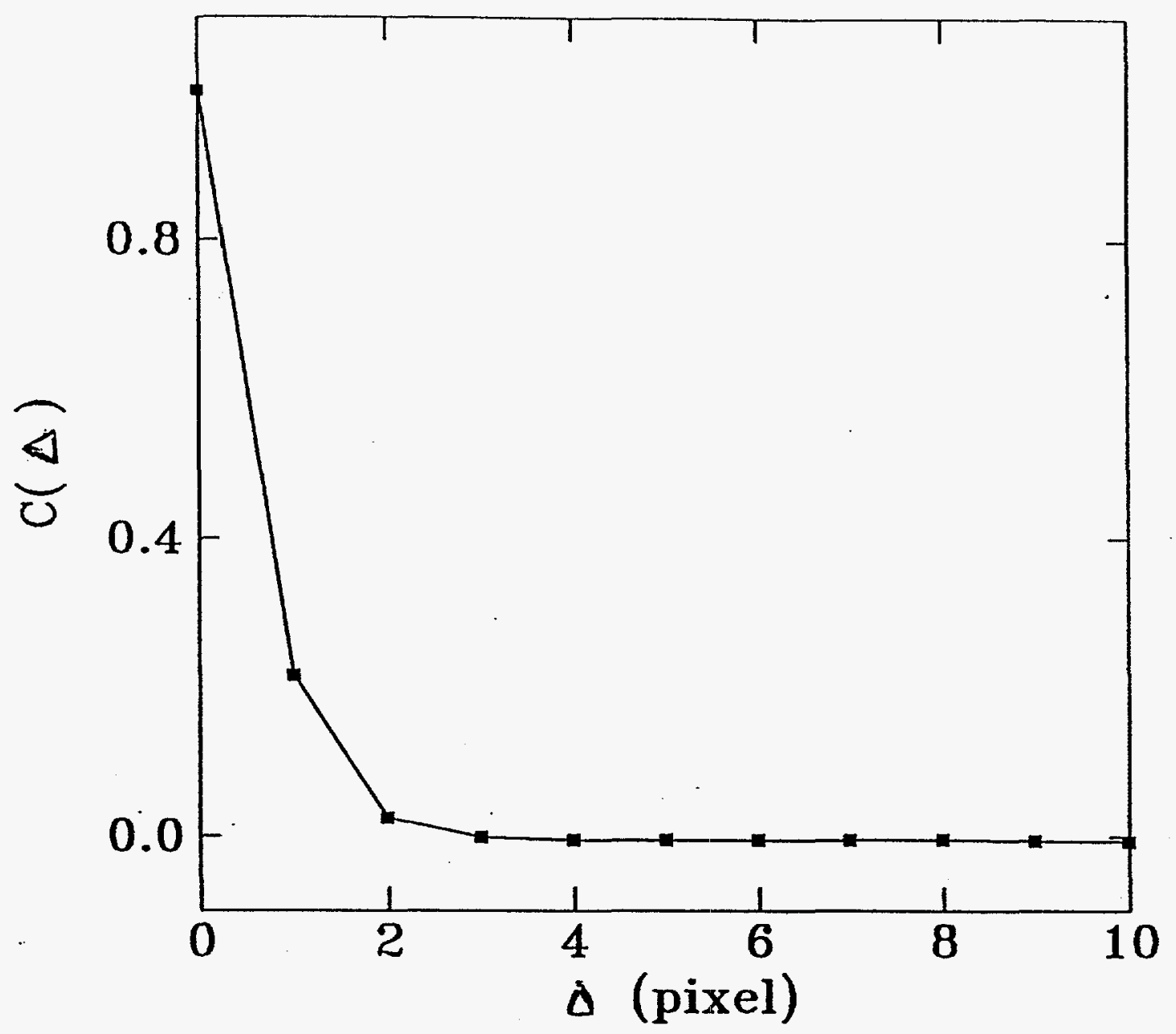

fig 2 
$7 \mathrm{keV}$ photons

CCD array or scintillator

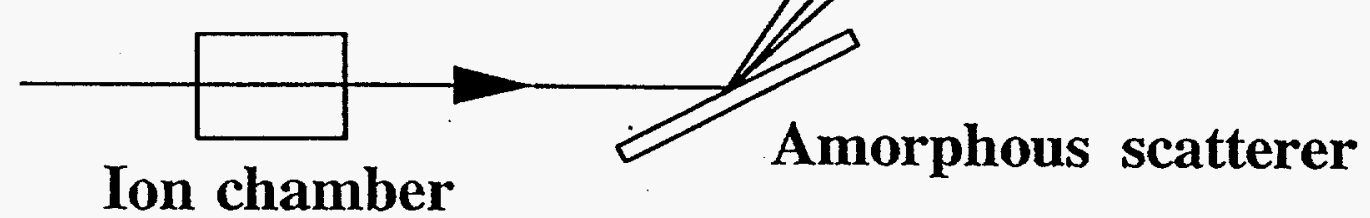




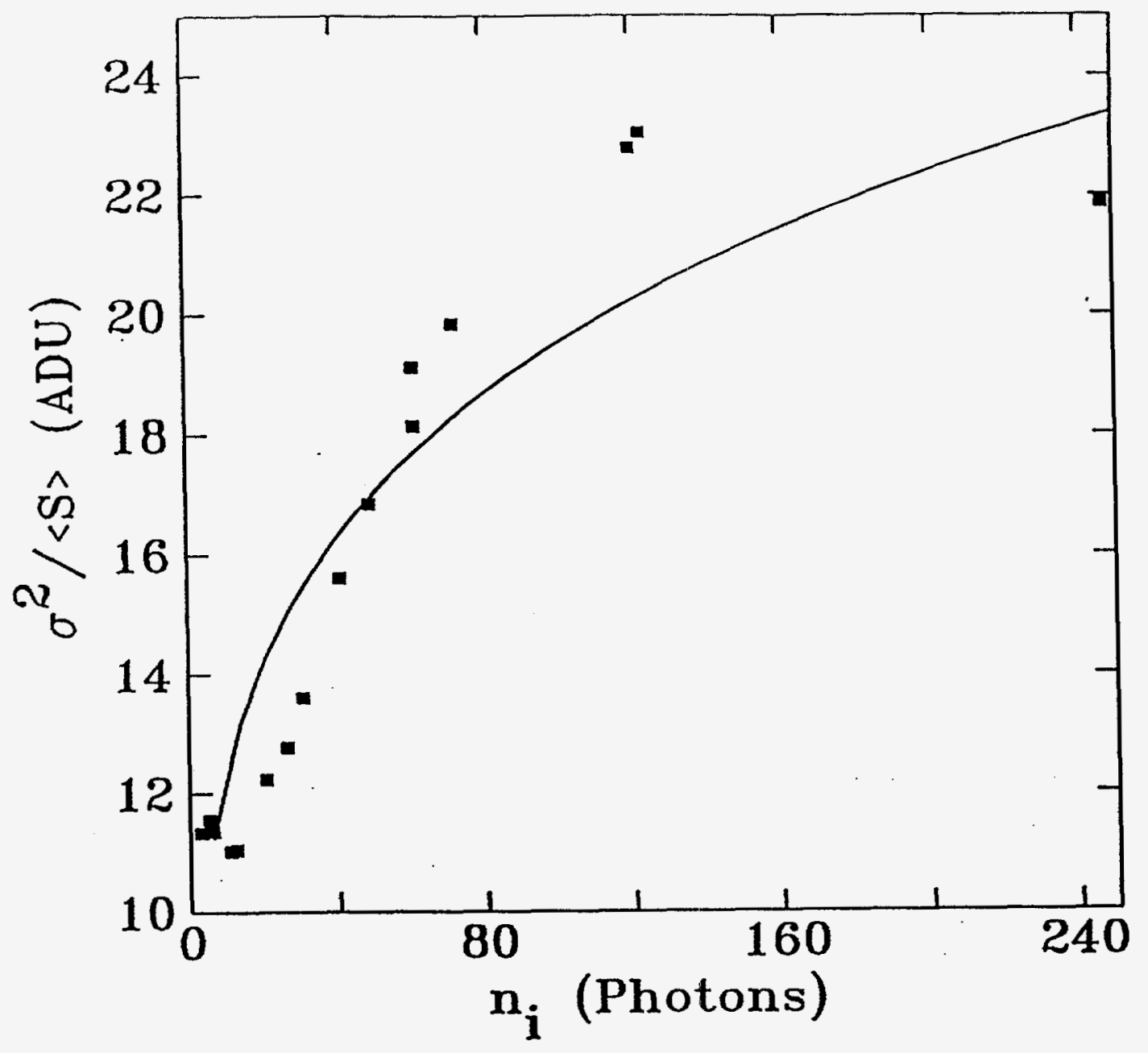

figh 


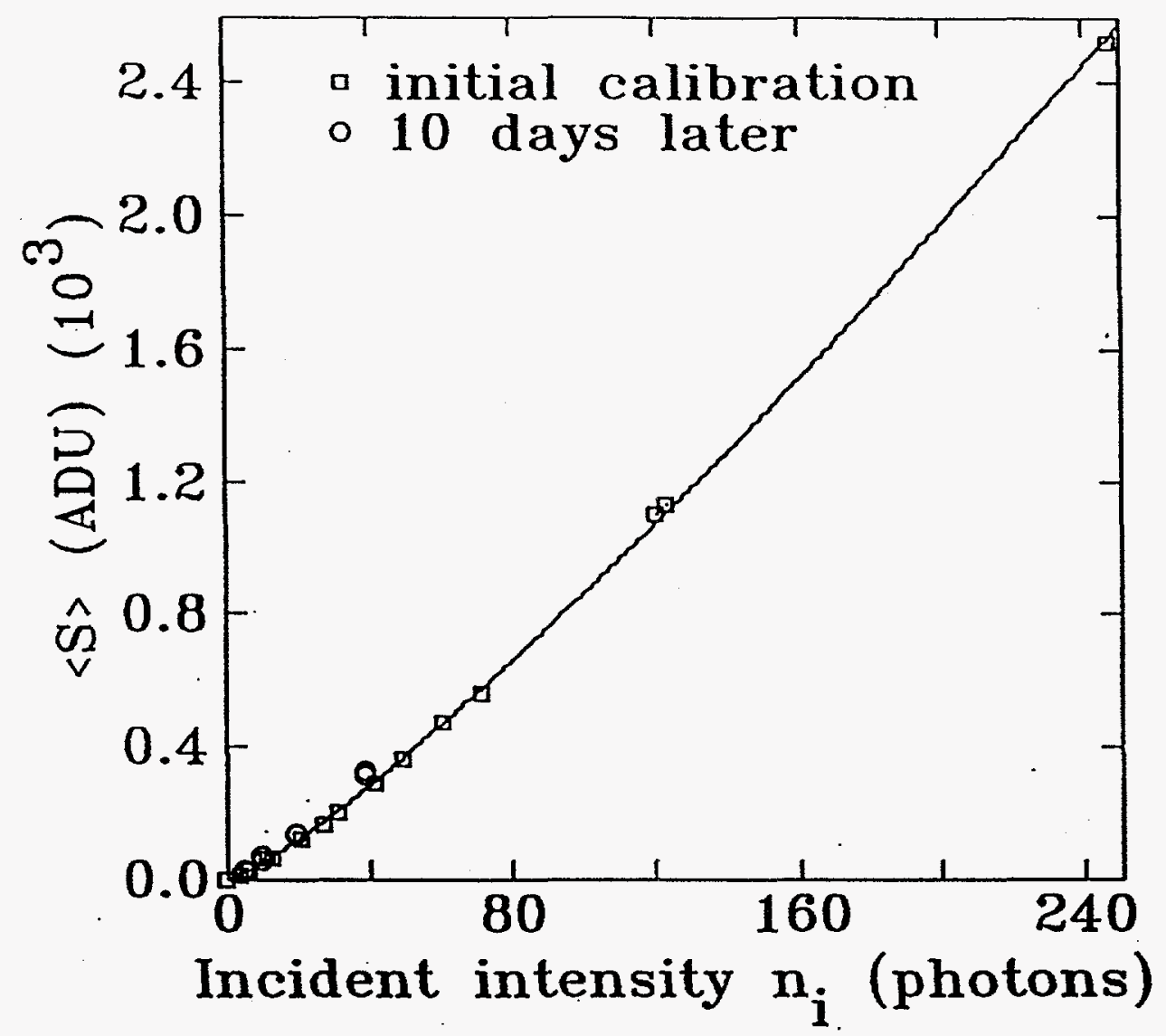




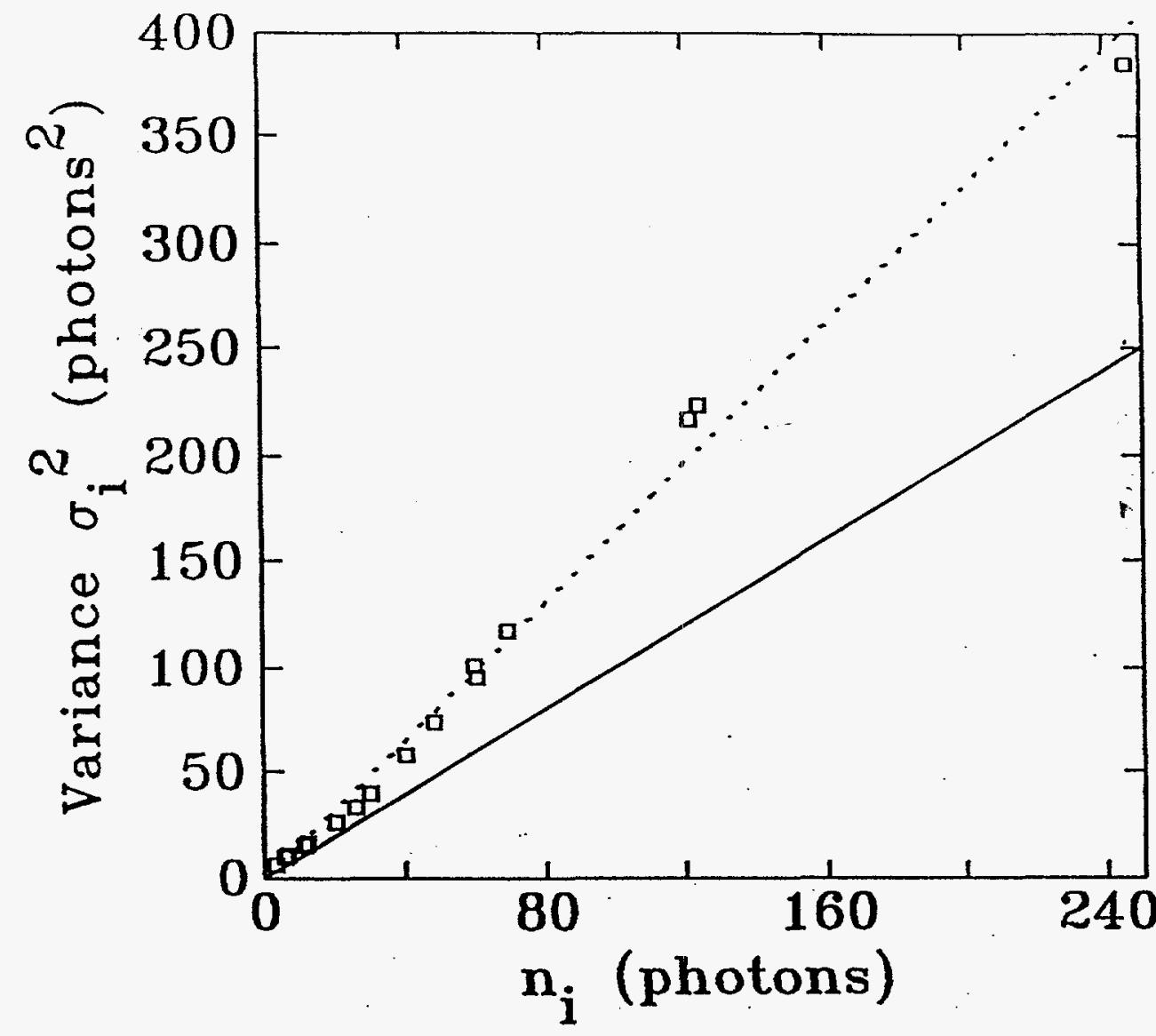




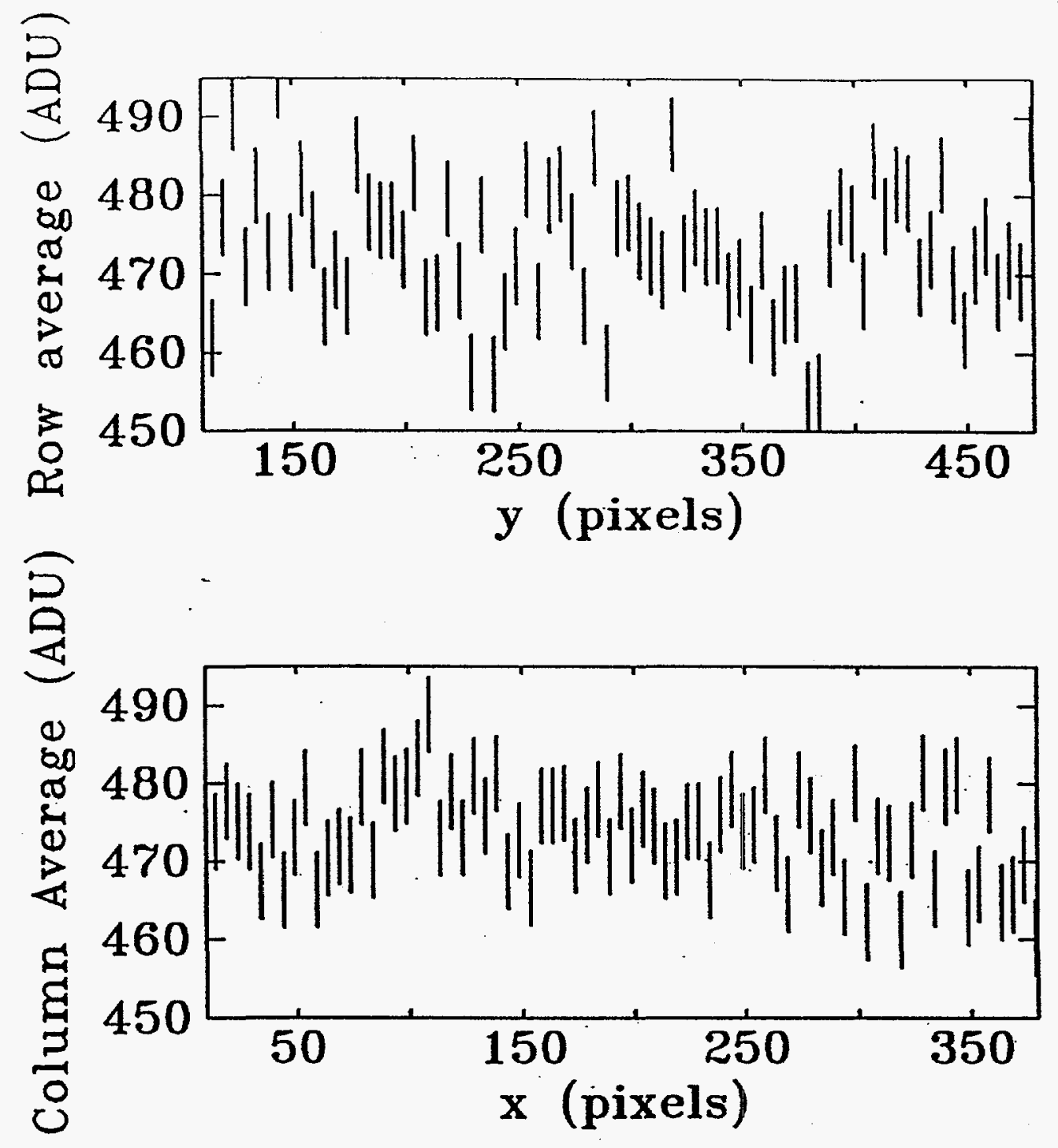



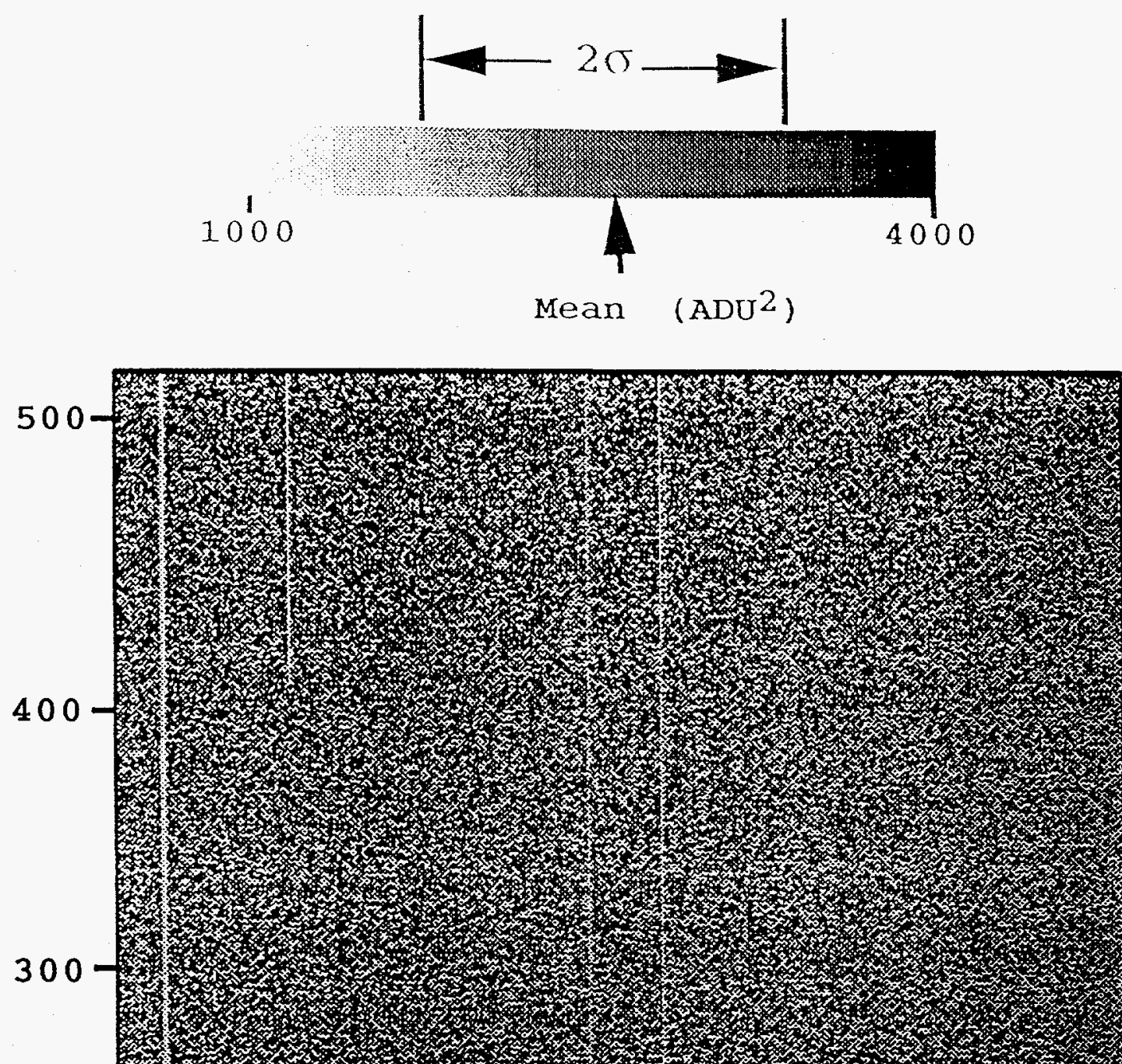

$y$ (pixels)

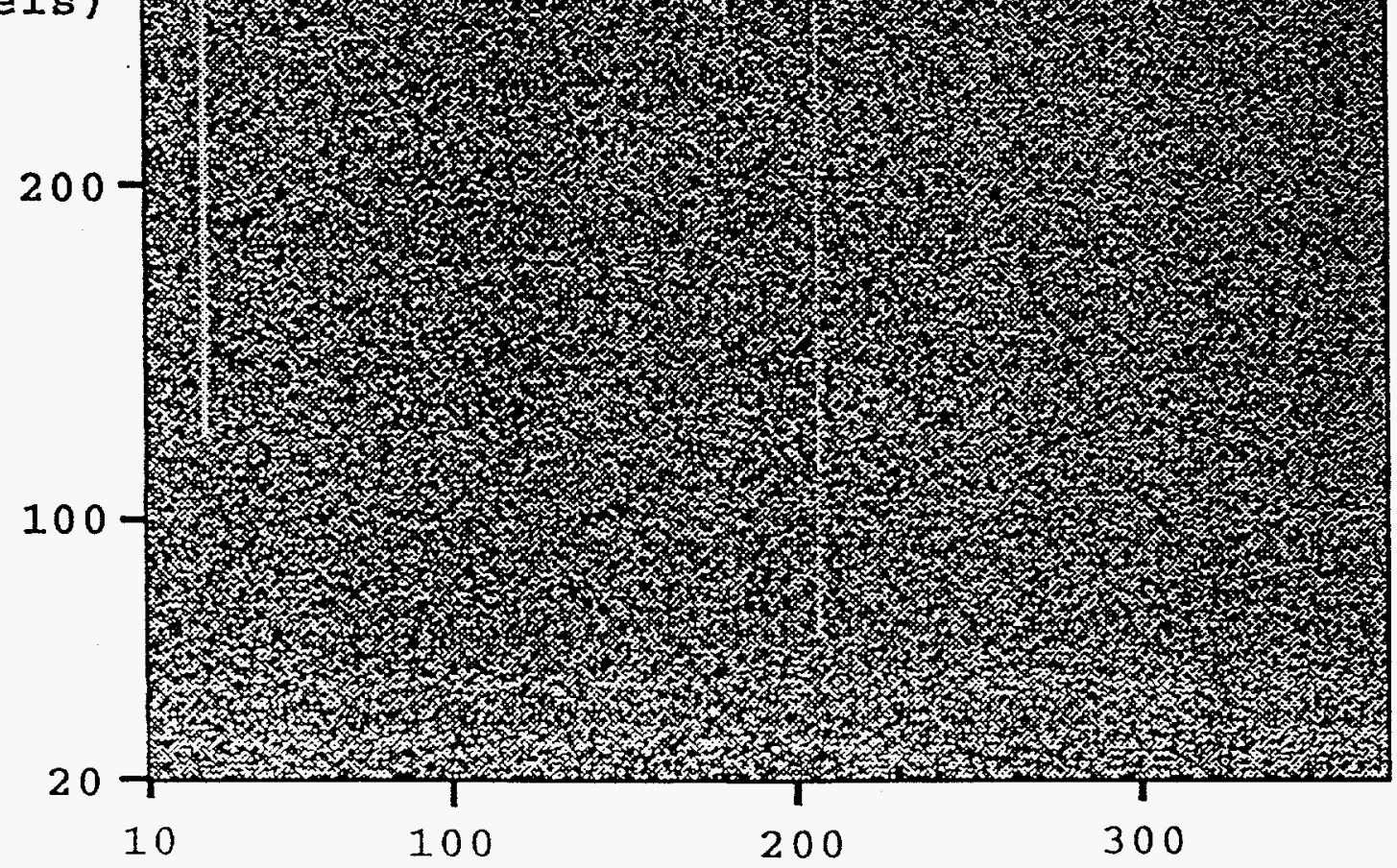

x (pixels) 


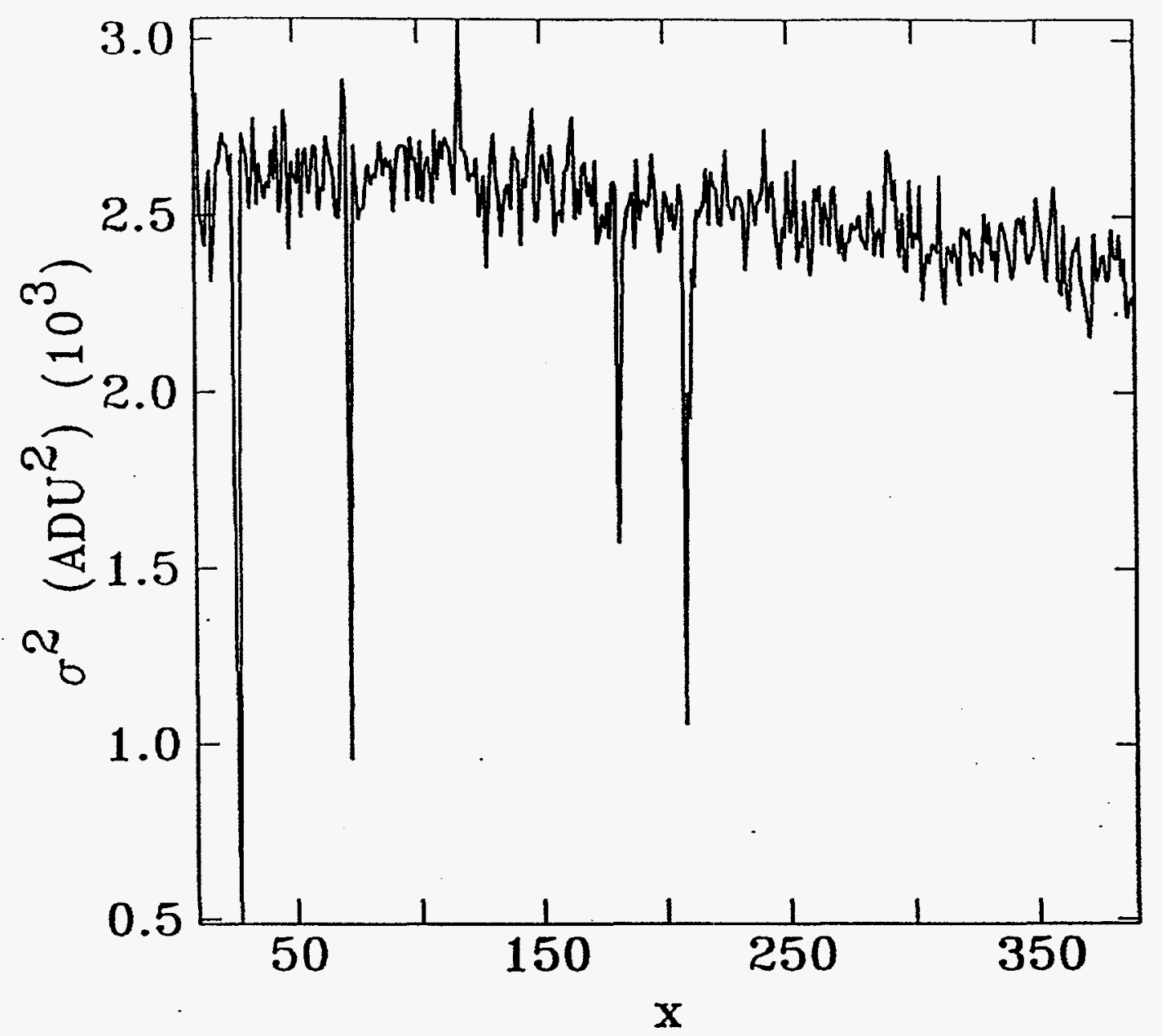

figg 


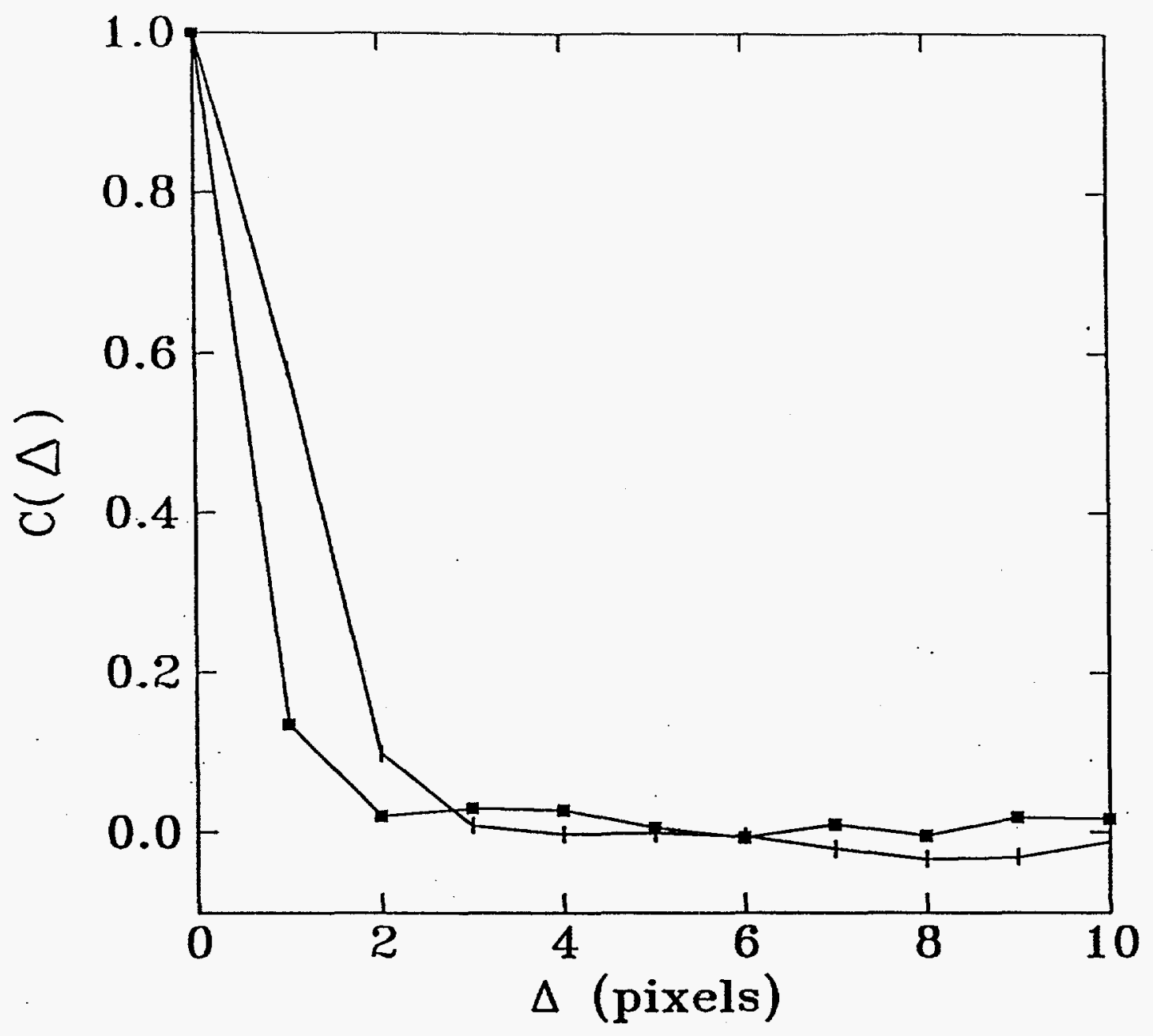

figro 